\title{
Some Special Associated Curves of Non-degenerate Curve in Anti de Sitter 3-Space
}

\author{
Mahmut Mak* and Hasan Altınbaş
}

(Communicated by Bülent ALTUNKAYA)

\begin{abstract}
In this paper, we investigate special associated curve of a non-degenerate Frenet curve according to the Sabban frame in anti de Sitter 3-space. Moreover, we give a construction method of Sabban apparatus of a special direction curve in terms of the elements of Sabban apparatus of its donor curve. Furthermore, we obtain some results for the direction curve with respect to special cases of the base curve. Finally, we give an example of a helix and its direction curve which is also a helix and draw theirs images under the stereographic projection in Minkowski 3-space.
\end{abstract}

Keywords: Frenet curve; associated curve; direction curve; donor curve; helix.

AMS Subject Classification (2010): Primary: 53A35 ; Secondary: $53 C 25$

*Corresponding author

\section{Introduction}

It is known that there are three kinds of Lorentzian space forms which is Minkowski 3-space, Anti de Sitter 3-space and de Sitter 3-space. Especially, Anti de Sitter 3-space is quite different from those of Minkowski space and de Sitter space according to causality.

The theory of the associated curve of a given curve has been studied by many authors in different ambient space from various viewpoints. For instance, in Euclidean 3-space, new associated curve was defined by Choi and Kim [3]. They introduced direction(i.e. special associated) curves of any base curve and give characterizations for the general and slant helices via their associated curves. After, in Minkowski 3-space, Choi et al. [4] studied some associated curves of a given Frenet curve to classify the general helices and the slant helices. Macit and Düldül defined some new associated curves ( $W$-direction, $W$-rectifying, $V$-direction) of a Frenet curve in Euclidean 3-space and Euclidean 4-space [8]. Kızıltuğ and Önder gave definition of associated curves of a Frenet curve in a three dimensional compact Lie group G [6]. They introduced the principal normal direction curve and principal normal donor curve and gave some characterizations for these curves in $\mathrm{G}$.

This paper is organized as follows. In section 2, we give local differential geometry of non-degenerate regular curves in Anti de Sitter 3-space and de Sitter 3-space with index two, which are denoted by $\mathbb{H}_{1}^{3}$ and $\mathbb{S}_{2}^{3}$, respectively. We call that a curve is AdS (dS) curve if the curve is immersed unit speed non-degenerate curve in $\mathbb{H}_{1}^{3}\left(\mathbb{S}_{2}^{3}\right)$. In section 3, we consider the concept of associated curve theory in $\mathbb{H}_{1}^{3}$. Let $W$ be a non-null unit vector field along the Frenet AdS curve $\gamma$ in $\mathbb{H}_{1}^{3}$. After we define non-degenerate $W$-direction curve $\widetilde{\gamma}$ of $\gamma$ which is called $W$-donor curve of $\widetilde{\gamma}$. In here, we choose a $W$-direction curve $\widetilde{\gamma}$ which is a principal unit normal of $\gamma$ with respect to Sabban frame in $\mathbb{H}_{1}^{3}$. Moreover, we give the relationship between the curves $\gamma$ and $\widetilde{\gamma}$ with respect to theirs Sabban apparatus. After, we obtain some corollaries for the associated curve $\widetilde{\gamma}$ when the curve $\gamma$ is a planar curve, horocycle, helix, respectively. In section 4 , we give definition of stereographic projection in $\mathbb{H}_{1}^{3}$ and $\mathbb{S}_{2}^{3}$. Then, we give an example

Received : 03-April-2017, Accepted : 08-September-2017 
for base curve and its special direction curve, which are helices. Finally, we draw the theirs images by using the stereographic projection in Minkowski 3-space.

\section{Preliminary}

In this section, we give the basic theory of local differential geometry of non-degenerate curves in de Sitter 3-space with index 2 and Anti de Sitter 3-space. For more detail and background about these spaces, see [2, 10].

Let $\mathbb{R}_{2}^{4}$ denote the four-dimensional semi Euclidean space with index two, that is, the real vector space $\mathbb{R}^{4}$ endowed with the scalar product

$$
\langle\boldsymbol{x}, \boldsymbol{y}\rangle=-x_{1} y_{1}-x_{2} y_{2}+x_{3} y_{3}+x_{4} y_{4}
$$

for all $\boldsymbol{x}=\left(x_{1}, x_{2}, x_{3}, x_{4}\right), \boldsymbol{y}=\left(y_{1}, y_{2}, y_{3}, y_{4}\right) \in \mathbb{R}^{4}$. Let $\left\{\boldsymbol{e}_{1}, \boldsymbol{e}_{2}, \boldsymbol{e}_{3}, \boldsymbol{e}_{4}\right\}$ be pseudo-orthonormal basis for $\mathbb{R}_{2}^{4}$. Then $\delta_{i j}$ is Kronecker-delta function such that $\left\langle\boldsymbol{e}_{i}, \boldsymbol{e}_{j}\right\rangle=\delta_{i j} \varepsilon_{j}$ for $\varepsilon_{1}=\varepsilon_{2}=-1, \varepsilon_{3}=\varepsilon_{4}=1$.

A vector $\boldsymbol{x} \in \mathbb{R}_{2}^{4}$ is called spacelike, timelike and lightlike (null) if $\langle\boldsymbol{x}, \boldsymbol{x}\rangle>0$ (or $\boldsymbol{x}=0$ ), $\langle\boldsymbol{x}, \boldsymbol{x}\rangle\langle 0$ and $\langle\boldsymbol{x}, \boldsymbol{x}\rangle=0$, respectively. The norm of a vector $\boldsymbol{x} \in \mathbb{R}_{2}^{4}$ is defined by $\|\boldsymbol{x}\|=\sqrt{|\langle\boldsymbol{x}, \boldsymbol{x}\rangle|}$. The signature of a vector $\boldsymbol{x}$ is denoted by

$$
\operatorname{sign}(\boldsymbol{x})=\left\{\begin{array}{cl}
1, & \boldsymbol{x} \text { is spacelike } \\
0, & \boldsymbol{x} \text { is null } \\
-1, & \boldsymbol{x} \text { is timelike }
\end{array}\right.
$$

The sets

$$
\begin{aligned}
\mathbb{S}_{2}^{3} & =\left\{\boldsymbol{x} \in \mathbb{R}_{2}^{4} \mid\langle\boldsymbol{x}, \boldsymbol{x}\rangle=1\right\} \\
\mathbb{H}_{1}^{3} & =\left\{\boldsymbol{x} \in \mathbb{R}_{2}^{4} \mid\langle\boldsymbol{x}, \boldsymbol{x}\rangle=-1\right\}
\end{aligned}
$$

are called de Sitter 3-space with index 2 (unit pseudosphere with dimension 3 and index 2 in $\mathbb{R}_{2}^{4}$ ) and Anti de Sitter 3 -space (unit pseudohyperbolic space with dimension 3 and index 2 in $\mathbb{R}_{2}^{4}$ ), respectively.

The pseudo vector product of vectors $\boldsymbol{x}, \boldsymbol{y}$ and $\boldsymbol{z}$ is given by

$$
\boldsymbol{x} \wedge \boldsymbol{y} \wedge \boldsymbol{z}=\left|\begin{array}{cccc}
-\boldsymbol{e}_{1} & -\boldsymbol{e}_{2} & \boldsymbol{e}_{3} & \boldsymbol{e}_{4} \\
x_{1} & x_{2} & x_{3} & x_{4} \\
y_{1} & y_{2} & y_{3} & y_{4} \\
z_{1} & z_{2} & z_{3} & z_{4}
\end{array}\right|
$$

where $\left\{\boldsymbol{e}_{1}, \boldsymbol{e}_{2}, \boldsymbol{e}_{3}, \boldsymbol{e}_{4}\right\}$ is the canonical basis of $\mathbb{R}_{2}^{4}$ and $\boldsymbol{x}=\left(x_{1}, x_{2}, x_{3}, x_{4}\right), \boldsymbol{y}=\left(y_{1}, y_{2}, y_{3}, y_{4}\right), \boldsymbol{z}=\left(z_{1}, z_{2}, z_{3}, z_{4}\right) \in \mathbb{R}_{2}^{4}$. Also, it is clear that

$$
\langle\boldsymbol{w}, \boldsymbol{x} \wedge \boldsymbol{y} \wedge \boldsymbol{z}\rangle=\operatorname{det}(\boldsymbol{w}, \boldsymbol{x}, \boldsymbol{y}, \boldsymbol{z})
$$

for any $\boldsymbol{w} \in \mathbb{R}_{2}^{4}$. Therefore, $\boldsymbol{x} \wedge \boldsymbol{y} \wedge \boldsymbol{z}$ is pseudo-orthogonal to each of the vectors $\boldsymbol{x}, \boldsymbol{y}$ and $\boldsymbol{z}$.

Unless otherwise stated for the sake of brevity, we will use the notation $\mathbb{M}^{3}\left(\delta_{0}\right)$ instead of the symbols $\mathbb{S}_{2}^{3}$ or $\mathbb{H}_{1}^{3}$. In here, if $\delta_{0}=1$ or $\delta_{0}=-1$, then $\mathbb{M}^{3}(1)=\mathbb{S}_{2}^{3}$ or $\mathbb{M}^{3}(-1)=\mathbb{H}_{1}^{3}$, respectively. Now, we give the basic theory of non-degenerate curves in $\mathbb{M}^{3}\left(\delta_{0}\right)$.

Let $\gamma: I \rightarrow \mathbb{M}^{3}\left(\delta_{0}\right)$ be regular curve (i.e., an immersed curve) for open subset $I \subset \mathbb{R}$ where $\operatorname{sign}(\gamma(t))=\delta_{0}$ for every $t \in I$. The regular curve $\gamma$ is said to be spacelike or timelike if $\gamma^{\prime}$ is a spacelike or timelike vector at any $t \in I$ where $\gamma^{\prime}(t)=d \gamma / d t$. The such curves are called non-degenerate curve. Since $\gamma$ is a non-degenerate curve, it admits an arc length parametrization $s=s(t)$. Thus, we can assume that $\gamma(s)$ is a unit speed curve. Then the unit tangent vector of $\gamma$ is given by $\boldsymbol{t}(s)=\gamma^{\prime}(s)$. Since $\langle\gamma(s), \gamma(s)\rangle=\delta_{0}$, we have $\left\langle\gamma(s), \boldsymbol{t}^{\prime}(s)\right\rangle=-\delta_{1}$ where $\delta_{1}=\operatorname{sign}(\boldsymbol{t}(s))$. The vector $\boldsymbol{t}^{\prime}(s)+\delta_{0} \delta_{1} \gamma(s)$ is pseudo-orthogonal to $\gamma(s)$ and $\boldsymbol{t}(s)$. In the case when $\left\langle\gamma^{\prime \prime}(s), \gamma^{\prime \prime}(s)\right\rangle \neq \delta_{0}$ and $\boldsymbol{t}^{\prime}(s)+\delta_{0} \delta_{1} \gamma(s) \neq 0$, the principle normal vector and the binormal vector of $\gamma$ is given by $\boldsymbol{n}(s)=\frac{\boldsymbol{t}^{\prime}(s)+\delta_{0} \delta_{1} \gamma(s)}{\left\|\boldsymbol{t}^{\prime}(s)+\delta_{0} \delta_{1} \gamma(s)\right\|}$ and $\boldsymbol{b}(s)=\gamma(s) \wedge \boldsymbol{t}(s) \wedge \boldsymbol{n}(s)$, respectively. Also, geodesic curvature of $\gamma$ are defined by $\kappa_{g}(s)=\left\|\boldsymbol{t}^{\prime}(s)+\delta_{0} \delta_{1} \gamma(s)\right\|$. Hence, we have pseudo-orthonormal frame field $\{\gamma(s), \boldsymbol{t}(s), \boldsymbol{n}(s), \boldsymbol{b}(s)\}$ of $\mathbb{R}_{2}^{4}$ along $\gamma$. The frame is also called the Sabban frame of non-degenerate curve $\gamma$ on $\mathbb{M}^{3}\left(\delta_{0}\right)$ such that

$$
\left\{\begin{array}{ccc}
\boldsymbol{t}(s) \wedge \boldsymbol{n}(s) \wedge \boldsymbol{b}(s) & = & -\delta_{0} \delta_{3} \gamma(s) \\
\boldsymbol{n}(s) \wedge \boldsymbol{b}(s) \wedge \gamma(s) & = & \delta_{1} \delta_{3} \boldsymbol{t}(s) \\
\boldsymbol{b}(s) \wedge \gamma(s) \wedge \boldsymbol{t}(s) & = & -\delta_{2} \delta_{3} \boldsymbol{n}(s) \\
\gamma(s) \wedge \boldsymbol{t}(s) \wedge \boldsymbol{n}(s) & = & \boldsymbol{b}(s)
\end{array}\right.
$$


where

$$
\left\{\begin{array}{c}
\operatorname{sign}(\boldsymbol{t}(s))=\delta_{1}, \operatorname{sign}(\boldsymbol{n}(s))=\delta_{2}, \operatorname{sign}(\boldsymbol{b}(s))=\delta_{3}, \\
\operatorname{det}(\gamma, \boldsymbol{t}, \boldsymbol{n}, \boldsymbol{b})=-\delta_{3} .
\end{array}\right.
$$

Now, if the assumption is $\left\langle\gamma^{\prime \prime}(s), \gamma^{\prime \prime}(s)>\neq \delta_{0}\right.$, we can give the following Frenet-Serret formulas of $\gamma$ such that

$$
\left[\begin{array}{c}
\gamma^{\prime}(s) \\
\boldsymbol{t}^{\prime}(s) \\
\boldsymbol{n}^{\prime}(s) \\
\boldsymbol{b}^{\prime}(s)
\end{array}\right]=\left[\begin{array}{cccc}
0 & 1 & 0 & 0 \\
-\delta_{0} \delta_{1} & 0 & \kappa_{g}(s) & 0 \\
0 & -\delta_{1} \delta_{2} \kappa_{g}(s) & 0 & -\delta_{1} \delta_{3} \tau_{g}(s) \\
0 & 0 & \delta_{1} \delta_{2} \tau_{g}(s) & 0
\end{array}\right]\left[\begin{array}{c}
\gamma(s) \\
\boldsymbol{t}(s) \\
\boldsymbol{n}(s) \\
\boldsymbol{b}(s)
\end{array}\right]
$$

where the geodesic torsion of $\gamma$ is given by $\tau_{g}(s)=\frac{\delta_{1} \operatorname{det}\left(\gamma(s), \gamma^{\prime}(s), \gamma^{\prime \prime}(s), \gamma^{\prime \prime \prime}(s)\right)}{\left(\kappa_{g}(s)\right)^{2}}$.

Remark 2.1. The condition $\left\langle\gamma^{\prime \prime}(s), \gamma^{\prime \prime}(s)>\neq \delta_{0}\right.$ is equivalent to $\kappa_{g}(s) \neq 0$. Moreover, we can show that $\kappa_{g}(s)=0$ and $\boldsymbol{t}^{\prime}(s)+\delta_{0} \delta_{1} \gamma(s)=0$ if and only if the non-degenerate curve $\gamma$ is a geodesic in $\mathbb{M}^{3}\left(\delta_{0}\right)$.

We will give the following definitions in $[1,2]$ and also they are valid for $\mathbb{S}_{2}^{3}$.

Definition 2.1. Let $\gamma: I \subset \mathbb{R} \rightarrow \mathbb{M}^{3}\left(\delta_{0}\right)$ is an immersed spacelike (timelike) curve according to the Sabban frame $\{\gamma, \boldsymbol{t}, \boldsymbol{n}, \boldsymbol{b}\}$ with geodesic curvature $\kappa_{g}$ and geodesic torsion $\tau_{g}$. Then,

- If $\tau_{g} \equiv 0, \gamma$ is called a planar curve in $\mathbb{M}^{3}\left(\delta_{0}\right)$.

- If $\kappa_{g} \equiv 1$ and $\tau_{g} \equiv 0, \gamma$ is called a horocycle in $\mathbb{M}^{3}\left(\delta_{0}\right)$.

- If $\kappa_{g}$ and $\tau_{g}$ are both non-zero constant, $\gamma$ is called a helix in $\mathbb{M}^{3}\left(\delta_{0}\right)$.

Remark 2.2. From now on, we call that $\gamma$ is a non-degenerate $A d S(d S)$ curve if $\gamma$ is an immersed non-dejenerate unit speed curve in $\mathbb{H}_{1}^{3}\left(\mathbb{S}_{2}^{3}\right)$.

\section{Associated Curves of the Non-degenerate Curve in $\mathbb{H}_{1}^{3}$}

In $[3,4]$, the authors introduces the definition of $W$-direction curve and $W$-donor curve in Euclidean space and Minkowski space. In the following, we investigate this concepts in $\mathbb{H}_{1}^{3}$ from this point of view Lorentzian space forms by using method in [7].

Definition 3.1. The non-degenerate regular curve $\gamma$ with $\kappa_{g} \neq 0$ is called the Frenet curve in $\mathbb{H}_{1}^{3}$.

Let consider a Frenet AdS curve $\gamma=\gamma(s): I \subset \mathbb{R} \rightarrow \mathbb{H}_{1}^{3}$ with the Sabban apparatus $\left\{\gamma(s), \boldsymbol{t}(s), \boldsymbol{n}(s), \boldsymbol{b}(s), \kappa_{g}(s), \tau_{g}(s)\right\}$ with the equations (2.3) and a non-null unit vector field $W \in \mathfrak{X}\left(\mathbb{H}_{1}^{3}\right)$ along the curve $\gamma$ be given by

$$
W(s)=c_{1}(s) \boldsymbol{t}(s)+c_{2}(s) \boldsymbol{n}(s)+c_{3}(s) \boldsymbol{b}(s)
$$

where $c_{i}(s)$ is arbitrary differentiable function and $s$ is the arc length parameter of $\gamma$. Since $W$ is non-null unit vector field in $\mathfrak{X}\left(\mathbb{H}_{1}^{3}\right)$, we have

$$
\sum_{i=1}^{3} \delta_{i} c_{i}^{2}= \pm 1, \quad c_{i}=c_{i}(s) .
$$

Definition 3.2. Let $\gamma$ be a Frenet AdS curve and $W$ be a non-null unit vector field along the curve $\gamma$ which is satisfying the equations (3.1) and (3.2). If there exist a curve $\widetilde{\gamma}$ with the Sabban apparatus $\left\{\widetilde{\gamma}, \widetilde{\boldsymbol{t}}, \widetilde{\boldsymbol{n}}, \widetilde{\boldsymbol{b}}, \widetilde{\kappa_{g}}, \widetilde{\tau_{g}}\right\}$ in $\mathbb{M}^{3}\left(\widetilde{\delta}_{0}\right)$ which is an integral curve of $W$ (i.e. $\widetilde{\boldsymbol{t}}=W$ ), then $\widetilde{\gamma}$ is called W-direction curve of $\gamma$ and $\gamma$ is called W-donor curve of $\widetilde{\gamma}$.

Thus, we can give the following associated curves of $\gamma$ in $\mathbb{H}_{1}^{3}$.

- If $W=\boldsymbol{t}$, then $\boldsymbol{t}$-direction curve $\widetilde{\gamma}$ of the curve $\gamma$ is trivially $\gamma($ i.e. $\widetilde{\boldsymbol{t}}=\boldsymbol{t}$ ).

- If $W=\boldsymbol{n}$, then $\widetilde{\boldsymbol{t}}=\boldsymbol{n}$. In this case, $\boldsymbol{n}$-direction curve $\widetilde{\gamma}$ of the curve $\gamma$ is called the principal direction curve of $\gamma$ and so the curve $\gamma$ is called the principal donor curve of $\widetilde{\gamma}$. 
- If $W=\boldsymbol{b}$, then $\widetilde{\boldsymbol{t}}=\boldsymbol{b}$. In this case, $\boldsymbol{b}$ - direction curve $\widetilde{\gamma}$ of the curve $\gamma$ is called the binormal direction curve of $\gamma$ and so the curve $\gamma$ is called the binormal donor curve of $\widetilde{\gamma}$.

Now, we will define a special associated curve of $\gamma$ in $\mathbb{H}_{1}^{3}$.

Definition 3.3. If $W=\frac{n^{\prime}}{\left\|n^{\prime}\right\|}$, then $\widetilde{t}=\frac{n^{\prime}}{\left\|n^{\prime}\right\|}$. In this case, the curve $\widetilde{\gamma}$ is called $W$-direction curve of the curve $\gamma$.

Remark 3.1. From now on, we will take as $W=\frac{\boldsymbol{n}^{\prime}}{\left\|\boldsymbol{n}^{\prime}\right\|}$ for calculations, unless otherwise stated. Hence, we easily see that $\widetilde{\boldsymbol{t}}=W$ such that $W=\frac{-\delta_{1} \delta_{2} \kappa_{g} t-\delta_{1} \delta_{3} \tau_{g} b}{\sqrt{\delta_{1} \kappa_{g}^{2}+\delta_{3} \tau_{g}^{2}}}$ by (2.4) and so it must be defined by $\widetilde{\gamma}=\boldsymbol{n}$ is non-degenerate curve which is fully lying on $\mathbb{M}^{3}\left(\widetilde{\delta}_{0}\right)$ (i.e. $\mathbb{H}_{1}^{3}$ or $\mathbb{S}_{2}^{3}$ ).

Let the $W$-direction curve $\widetilde{\gamma}: J \rightarrow \mathbb{M}^{3}\left(\widetilde{\delta}_{0}\right)$ of $\gamma=\gamma(s)$ be defined by

$$
\widetilde{\gamma}(\tilde{s}(s))=\boldsymbol{n}(s)
$$

with the Sabban aparatus $\left\{\widetilde{\gamma}(\tilde{s}), \widetilde{\boldsymbol{t}}(\tilde{s}), \widetilde{\boldsymbol{n}}(\tilde{s}), \widetilde{\boldsymbol{b}}(\tilde{s}), \widetilde{\kappa_{g}}(\tilde{s}), \widetilde{\tau_{g}}(\tilde{s})\right\}$ where

$$
\operatorname{sign}(\widetilde{\gamma})=\widetilde{\delta}_{0}, \operatorname{sign}(\widetilde{\boldsymbol{t}})=\widetilde{\delta}_{1}, \operatorname{sign}(\widetilde{\boldsymbol{n}})=\widetilde{\delta}_{2}, \operatorname{sign}(\widetilde{\boldsymbol{b}})=\widetilde{\delta}_{3}
$$

and $\tilde{s}$ be the arc length parameter of $\widetilde{\gamma}$ such that $\tilde{s}: I \rightarrow J$ is a regular differentiable function. It is clearly that if the signature $\widetilde{\delta}_{0}$ is equal to -1 or 1 , then $\mathbb{M}^{3}(-1)$ is $\mathbb{H}_{1}^{3}$ or $\mathbb{S}_{2}^{3}$, respectively.

Now, differentiating both sides of (3.3) with respect to $s$ and by using (2.4), we get

$$
\frac{d \tilde{s}}{d s} \widetilde{\boldsymbol{t}}=-\delta_{1} \delta_{2} \kappa_{g} \boldsymbol{t}-\delta_{1} \delta_{3} \tau_{g} \boldsymbol{b}
$$

where

$$
\frac{d \tilde{s}}{d s}= \pm \sqrt{\widetilde{\delta}_{1}\left(\delta_{1} \kappa_{g}^{2}+\delta_{3} \tau_{g}^{2}\right)}
$$

with the condition

$$
\widetilde{\delta}_{1}\left(\delta_{1} \kappa_{g}^{2}+\delta_{3} \tau_{g}^{2}\right)>0 .
$$

Moreover, by using (3.3) and (3.6), we have the following signature table

\begin{tabular}{|c|c|c|c|}
\hline$\widetilde{\delta}_{0}=-1$ & $\widetilde{\delta}_{1}=1 ; \widetilde{\delta}_{2}=-\widetilde{\delta}_{3}$ & $\delta_{0}=-1$ & $\delta_{1}=\delta_{3}=1 ; \delta_{2}=-1$ \\
\hline \multirow{2}{*}{$\widetilde{\delta}_{0}=1$} & $\widetilde{\delta}_{1}=1 ; \widetilde{\delta}_{2}=\widetilde{\delta}_{3}=-1$ & \multirow{2}{*}{$\delta_{0}=-1$} & $\delta_{1}=-\delta_{3} ; \delta_{2}=1$ \\
\cline { 2 - 2 } & $\widetilde{\delta}_{1}=-1 ; \widetilde{\delta}_{2}=-\widetilde{\delta}_{3}$ & & \\
\hline
\end{tabular}

Table 1. Signature table for $\mathrm{W}$-direction curve and $\mathrm{W}$-donor curve

Before the calculations, we give the following corollaries from Table 1

Corollary 3.1. There exist no timelike $W$-direction curve $\widetilde{\gamma}$ in $\mathbb{H}_{1}^{3}$ of any non-degenerate curve $\gamma$ in $\mathbb{H}_{1}^{3}$.

Corollary 3.2. There exist no timelike $W$-donor curve $\gamma$ in $\mathbb{H}_{1}^{3}$ of any non-degenerate curve $\widetilde{\gamma}$ in $\mathbb{H}_{1}^{3}$.

Now, we will give a construction method of Sabban apparatus of $\widetilde{\gamma}$ in terms of the elements of Sabban apparatus of its W-donor curve $\gamma$.

Theorem 3.1. Let $\widetilde{\gamma}=\widetilde{\gamma}(\tilde{s})$ be W-direction curve in $\mathbb{M}^{3}\left(\widetilde{\delta_{0}}\right)$ of any non-degenerate AdS curve $\gamma=\gamma(s)$ in $\mathbb{H}_{1}^{3}$. Then the Sabban apparatus of $W$-direction curve $\widetilde{\gamma}$ can be constructed with according to the elements of Sabban apparatus of $\gamma$ for all possible cases from the Table 1.

Proof. If $\boldsymbol{n}$ is timelike(spacelike), then $\widetilde{\gamma}$ is fully lying in $\mathbb{H}_{1}^{3}\left(\mathbb{S}_{2}^{3}\right)$ by (3.3). Thus, we have the following two main cases by using Table 1. 
Case 1: $\left(\widetilde{\delta}_{0}=-1 ; \widetilde{\delta}_{1}=1, \widetilde{\delta}_{2}=-\widetilde{\delta}_{3} ; \delta_{0}=-1 ; \delta_{1}=\delta_{3}=1, \delta_{2}=-1\right)$

In this case, $\widetilde{\gamma}$ is spacelike W-direction curve of the spacelike curve $\gamma$ in $\mathbb{H}_{1}^{3}$. Without loss of generality, we choice positive sign of $\frac{d \hat{s}}{d s}$. By using (3.4) and (3.5), we have

$$
\frac{d \tilde{s}}{d s}=\sqrt{\kappa_{g}^{2}+\tau_{g}^{2}}
$$

and

$$
\widetilde{\boldsymbol{t}}=\cos \theta_{1} \boldsymbol{t}+\sin \theta_{1} \boldsymbol{b}, \theta_{1}=\theta_{1}(s)
$$

where

$$
\cos \theta_{1}=\frac{\kappa_{g}}{\sqrt{\kappa_{g}^{2}+\tau_{g}^{2}}}, \sin \theta_{1}=\frac{-\tau_{g}}{\sqrt{\kappa_{g}^{2}+\tau_{g}^{2}}}, \tan ^{-1}\left(\theta_{1}\right)=\frac{-\kappa_{g}}{\tau_{g}} .
$$

By using (2.4),(3.3),(3.7) and (3.8),

$$
\widetilde{\boldsymbol{n}}=\frac{\cos \theta_{1}}{\sqrt{\left.\mid \theta_{1}{ }^{2}\right)^{2}-\cos ^{2} \theta_{1} \mid}} \gamma-\frac{\theta_{1}{ }^{\prime}}{\sqrt{\left|\left(\theta_{1}{ }^{\prime}\right)^{2}-\cos ^{2} \theta_{1}\right|}}\left(\sin \theta_{1} \boldsymbol{t}-\cos \theta_{1} \boldsymbol{b}\right) .
$$

We have two possible subcases of the Case 1 according to the signature $\widetilde{\delta}_{2}$ of $\widetilde{\boldsymbol{n}}$.

Subcase 1.1: $\left(\widetilde{\delta}_{2}=1 ; \widetilde{\delta}_{3}=-1\right)$

In this subcase, $\widetilde{\boldsymbol{n}}$ is spacelike so $\widetilde{\boldsymbol{b}}$ must be timelike such that $\left(\theta_{1}{ }^{\prime}\right)^{2}-\cos ^{2} \theta_{1}>0$. Then, we get

$$
\widetilde{\boldsymbol{n}}=\left(\sinh \varphi_{1}\right) \gamma+\cosh \varphi_{1}\left(\sin \theta_{1} \boldsymbol{t}-\cos \theta_{1} \boldsymbol{b}\right), \varphi_{1}=\varphi_{1}(s)
$$

where

$$
\sinh \varphi_{1}=\frac{\cos \theta_{1}}{\sqrt{\left(\theta_{1}^{\prime}\right)^{2}-\cos ^{2} \theta_{1}}}, \cosh \varphi_{1}=\frac{-\theta_{1}{ }^{\prime}}{\sqrt{\left(\theta_{1}^{\prime}\right)^{2}-\cos ^{2} \theta_{1}}}
$$

Hence, we get easily

$$
\widetilde{\boldsymbol{b}}=\left(\cosh \varphi_{1}\right) \gamma+\sinh \varphi_{1}\left(\sin \theta_{1} \boldsymbol{t}-\cos \theta_{1} \boldsymbol{b}\right)
$$

and

$$
\widetilde{\kappa_{g}}=\sqrt{\frac{\left(\theta_{1}^{\prime}\right)^{2}-\cos ^{2} \theta_{1}}{\kappa_{g}^{2}+\tau_{g}^{2}}}, \widetilde{\tau_{g}}=\frac{1+\sin \theta_{1}}{\sqrt{\kappa_{g}^{2}+\tau_{g}^{2}}}
$$

by using (2.1),(2.2) and (2.4).

Subcase 1.2: $\left(\widetilde{\delta}_{2}=-1 ; \widetilde{\delta}_{3}=1\right)$

In this subcase, $\widetilde{\boldsymbol{n}}$ is timelike so $\widetilde{\boldsymbol{b}}$ must be spacelike such that $\left(\theta_{1}{ }^{\prime}\right)^{2}-\cos ^{2} \theta_{1}<0$. Then, we obtain

$$
\widetilde{\boldsymbol{n}}=\left(\cosh \varphi_{2}\right) \gamma+\sinh \varphi_{2}\left(\sin \theta_{1} \boldsymbol{t}-\cos \theta_{1} \boldsymbol{b}\right), \varphi_{2}=\varphi_{2}(s)
$$

where

$$
\cosh \varphi_{2}=\frac{\cos \theta_{1}}{\sqrt{\cos ^{2} \theta_{1}-\left(\theta_{1}^{\prime}\right)^{2}}}, \sinh \varphi_{2}=\frac{-\theta_{1}{ }^{\prime}}{\sqrt{\cos ^{2} \theta_{1}-\left(\theta_{1}^{\prime}\right)^{2}}}
$$

Thus, we have

$$
\widetilde{\boldsymbol{b}}=\left(\sinh \varphi_{2}\right) \gamma+\cosh \varphi_{2}\left(\sin \theta_{1} \boldsymbol{t}-\cos \theta_{1} \boldsymbol{b}\right)
$$

and

$$
\widetilde{\kappa_{g}}=\sqrt{\frac{\cos ^{2} \theta_{1}-\left(\theta_{1}^{\prime}\right)^{2}}{\kappa_{g}^{2}+\tau_{g}^{2}}}, \widetilde{\tau_{g}}=\frac{-\left(1+\sin \theta_{1}\right)}{\sqrt{\kappa_{g}^{2}+\tau_{g}^{2}}}
$$

by using (2.1),(2.2) and (2.4).

Now, if $\boldsymbol{n}$ is spacelike, then $\widetilde{\gamma}$ is fully lying in $\mathbb{S}_{2}^{3}$ by (3.3). Hence, we have the following the other possible cases. 
Case 2: $\left(\widetilde{\delta}_{0}=1 ; \delta_{0}=-1, \delta_{1}=-\delta_{3}, \delta_{2}=1\right)$

In this case, $\widetilde{\gamma}$ is spacelike or timelike $\mathrm{W}$-direction curve which is fully lying on $\mathbb{S}_{2}^{3}$ of the non-degenerate curve $\gamma$ which is fully lying on $\mathbb{H}_{1}^{3}$. Without loss of generality, we choice positive sign of $\frac{d \tilde{s}}{d s}$. By using (3.4) and (3.5), we have

$$
\frac{d \tilde{s}}{d s}=\sqrt{\tilde{\delta}_{1} \delta_{1}\left(\kappa_{g}^{2}-\tau_{g}^{2}\right)}
$$

under the condition

$$
\tilde{\delta}_{1} \delta_{1}\left(\kappa_{g}^{2}-\tau_{g}^{2}\right)>0
$$

and

$$
\widetilde{\boldsymbol{t}}=\cosh \xi_{1} \boldsymbol{t}+\sinh \xi_{1} \boldsymbol{b}, \xi_{1}=\xi_{1}(s)
$$

where

$$
\cosh \xi_{1}=\frac{-\delta_{1} \kappa_{g}}{\sqrt{\tilde{\delta}_{1} \delta_{1}\left(\kappa_{g}^{2}-\tau_{g}^{2}\right)}}, \sinh \xi_{1}=\frac{\tau_{g}}{\sqrt{\tilde{\delta}_{1} \delta_{1}\left(\kappa_{g}^{2}-\tau_{g}^{2}\right)}}, \tanh ^{-1}\left(\xi_{1}\right)=\frac{-\delta_{1} \kappa_{g}}{\tau_{g}} .
$$

By using (2.4),(3.3),(3.12) and (3.13), we get

$$
\widetilde{\boldsymbol{n}}=\frac{\delta_{1} \cosh \xi_{1}}{\sqrt{\left|\cosh ^{2} \xi_{1}+\delta_{1}\left(\xi_{1}\right)^{2}\right|}} \gamma+\frac{\xi_{1}{ }^{\prime}}{\sqrt{\left|\cosh ^{2} \xi_{1}+\delta_{1}\left(\xi_{1}\right)^{2}\right|}}\left(\sinh \xi_{1} \boldsymbol{t}-\cosh \xi_{1} \boldsymbol{b}\right) .
$$

Thus, we have two possible subcases of the Case 2 with according to the signatures $\widetilde{\boldsymbol{n}}$ and $\widetilde{\boldsymbol{b}}$.

Subcase 2.1: $\left(\widetilde{\delta}_{2}=1, \widetilde{\delta}_{1}=\widetilde{\delta}_{3}=-1 ; \delta_{1}=-1, \delta_{2}=\delta_{3}=1\right)$

In this subcase, $\widetilde{\boldsymbol{n}}$ is spacelike so $\widetilde{\boldsymbol{b}}$ must be timelike such that $\left(\xi_{1}{ }^{\prime}\right)^{2}-\cosh ^{2} \xi_{1}>0$. Then, we get

$$
\widetilde{\boldsymbol{n}}=\left(\sinh \eta_{1}\right) \gamma+\cosh \eta_{1}\left(\sinh \xi_{1} \boldsymbol{t}-\cosh \xi_{1} \boldsymbol{b}\right), \eta_{1}=\eta_{1}(s)
$$

where

$$
\sinh \eta_{1}=\frac{-\cosh \xi_{1}}{\sqrt{\left(\xi_{1}^{\prime}\right)^{2}-\cosh ^{2} \xi_{1}}}, \cosh \eta_{1}=\frac{\xi_{1}{ }^{\prime}}{\sqrt{\left(\xi_{1}^{\prime}\right)^{2}-\cosh ^{2} \xi_{1}}}
$$

Hence, we obtain

$$
\widetilde{\boldsymbol{b}}=\left(-\cosh \eta_{1}\right) \gamma-\sinh \eta_{1}\left(\sinh \xi_{1} \boldsymbol{t}-\cosh \xi_{1} \boldsymbol{b}\right)
$$

and

$$
\widetilde{\kappa_{g}}=\sqrt{\frac{\left(\xi_{1}^{\prime}\right)^{2}-\cosh ^{2} \xi_{1}}{\kappa_{g}^{2}-\tau_{g}^{2}}}, \widetilde{\tau_{g}}=\frac{1-\sinh \xi_{1}}{\sqrt{\kappa_{g}^{2}-\tau_{g}^{2}}}
$$

by straightforward calculations.

Subcase 2.2: $\left(\widetilde{\delta}_{1}=1, \widetilde{\delta}_{2}=\widetilde{\delta}_{3}=-1 ; \delta_{1}=\delta_{2}=1, \delta_{3}=-1\right)$

In this subcase, $\widetilde{\boldsymbol{n}}$ and $\widetilde{\boldsymbol{b}}$ must be timelike. Then, we get

$$
\widetilde{\boldsymbol{n}}=\left(\cos \eta_{2}\right) \gamma+\sin \eta_{2}\left(\sinh \xi_{1} \boldsymbol{t}-\cosh \xi_{1} \boldsymbol{b}\right), \eta_{2}=\eta_{2}(s)
$$

where

$$
\cos \eta_{2}=\frac{\cosh \xi_{1}}{\sqrt{\cosh ^{2} \xi_{1}+\left(\xi_{1}^{\prime}\right)^{2}}}, \sin \eta_{2}=\frac{\xi_{1}{ }^{\prime}}{\sqrt{\cosh ^{2} \xi_{1}+\left(\xi_{1}{ }^{\prime}\right)^{2}}}
$$

Consequently, we get

$$
\widetilde{\boldsymbol{b}}=\left(\sin \eta_{2}\right) \gamma-\cos \eta_{2}\left(\sinh \xi_{1} \boldsymbol{t}-\cosh \xi_{1} \boldsymbol{b}\right)
$$

and

$$
\widetilde{\kappa_{g}}=\sqrt{\frac{\cosh ^{2} \xi_{1}+\left(\xi_{1}^{\prime}\right)^{2}}{\kappa_{g}^{2}-\tau_{g}^{2}}}, \widetilde{\tau_{g}}=\frac{\sinh \xi_{1}-1}{\sqrt{\kappa_{g}^{2}-\tau_{g}^{2}}}
$$

by straightforward calculations. The proof is complete. 
Corollary 3.3. Let $\widetilde{\gamma}$ be the $W$-direction curve of the curve $\gamma$. Then the following statements are equivalent:

(i) $\gamma$ is helix in $\mathbb{H}_{1}^{3}$,

(ii) The angle between tangent vectors of $\gamma$ and $\widetilde{\gamma}$ at corresponding points (considered as vectors in $\mathbb{R}_{2}^{4}$ ) is constant,

(iii) The angle between binormal vectors of $\gamma$ and $\widetilde{\gamma}$ at corresponding points (considered as vectors in $\mathbb{R}_{2}^{4}$ ) is constant,

(iv) $\gamma$ is the W-direction curve of the curve $\widetilde{\gamma}$.

Proof. We see easily that the statements (i) and (ii) are equivalent by using the equations (3.7),(3.8) and (3.12),(3.13). $\theta_{1}$ and $\xi_{1}$ are constant by the equations (3.7) and (3.12), if the angle between tangent vectors is constant. Then we show that the angle between binormal vectors is constant from the equations (3.10),(3.11),(3.15) and (3.16) or vice versa. So the statements (ii) and (iii) are equivalent. Finally, the statements (iii) and (iv) are equivalent from the equations (3.9) and (3.14). The proof is complete.

Corollary 3.4. Let $\widetilde{\gamma}$ be the $W$-direction curve of the curve $\gamma$. Then the following statements are satisfied:

(i) If $\gamma$ is a planar curve or a horocycle in $\mathbb{H}_{1}^{3}$, then $\widetilde{\gamma}$ is a helix with $\widetilde{\kappa_{g}}=1$ and $\widetilde{\tau_{g}}=-1$ in $\mathbb{M}^{3}\left(\widetilde{\delta}_{0}\right)$,

(ii) If $\gamma$ is a helix in $\mathbb{H}_{1}^{3}$, then $\widetilde{\gamma}$ is a helix in $\mathbb{M}^{3}\left(\widetilde{\delta}_{0}\right)$,

\section{Stereographic Projection of $\mathbb{H}_{1}^{3}$ and $\mathbb{S}_{2}^{3}$}

Let $\mathbb{R}_{1}^{3}$ denote Minkowski 3-space (three-dimensional semi Euclidean space with index one), that is, the real vector space $\mathbb{R}^{3}$ endowed with the scalar product

$$
\langle\overline{\boldsymbol{x}}, \overline{\boldsymbol{y}}\rangle_{\star}=-\overline{x_{1}} \overline{y_{1}}+\overline{x_{2}} \overline{y_{2}}+\overline{x_{3}} \overline{y_{3}}
$$

for all $\overline{\boldsymbol{x}}=\left(\overline{x_{1}}, \overline{x_{2}}, \overline{x_{3}}\right), \overline{\boldsymbol{y}}=\left(\overline{y_{1}}, \overline{y_{2}}, \overline{y_{3}}\right) \in \mathbb{R}^{3}$. The set $\mathbb{S}_{1}^{2}=\left\{\overline{\boldsymbol{x}} \in \mathbb{R}_{1}^{3} \mid\langle\overline{\boldsymbol{x}}, \overline{\boldsymbol{x}}\rangle_{\star}=1\right\}$ is called de Sitter plane (unit pseudosphere with dimension 2 and index 1 in $\mathbb{R}_{1}^{3}$ ).

Definition 4.1. The conformal map

$$
\Phi: \mathbb{H}_{1}^{3} \backslash \Gamma \rightarrow \mathbb{R}_{1}^{3} \backslash \mathbb{S}_{1}^{2}, \Phi(\boldsymbol{x})=\left(\frac{x_{2}}{1+x_{1}}, \frac{x_{3}}{1+x_{1}}, \frac{x_{4}}{1+x_{1}}\right)
$$

is called the stereographic projection of $\mathbb{H}_{1}^{3}$ according to set $\Gamma=\left\{\boldsymbol{x} \in \mathbb{H}_{1}^{3} \mid x_{1}=-1\right\}$ [9].

Now, we give the stereographic projection of $\mathbb{S}_{2}^{3}$.

Lemma 4.1. The mapping

$$
\mu: \mathbb{R}_{2}^{3} \rightarrow \mathbb{R}_{1}^{3}, \mu\left(p_{1}, p_{2}, p_{3}\right)=\left(p_{3}, p_{1}, p_{2}\right)
$$

is an anti-isometry that carries each $\mathbb{S}_{1}^{2}$ anti-isometrically onto $\mathbb{H}_{1}^{2}$ and vice versa [10].

Definition 4.2. The map

$$
\Psi: \mathbb{S}_{2}^{3} \backslash \Lambda \rightarrow \mathbb{R}_{2}^{3} \backslash \mathbb{H}_{1}^{2}, \Psi(\mathbf{x})=\left(\frac{x_{1}}{1-x_{4}}, \frac{x_{2}}{1-x_{4}}, \frac{x_{3}}{1-x_{4}}\right)
$$

is called the stereographic projection of $\mathbb{S}_{2}^{3}$ according to set $\Lambda=\left\{\boldsymbol{x} \in \mathbb{S}_{2}^{3} \mid x_{4}=1\right\}$ [5].

Hence, we say that the following conformal map

$$
\mu \circ \Psi=\widetilde{\Psi}: \mathbb{S}_{2}^{3} \backslash \Lambda \rightarrow \mathbb{R}_{1}^{3} \backslash \mathbb{S}_{1}^{2}, \widetilde{\Psi}(\mathbf{x})=\left(\frac{x_{3}}{1-x_{4}}, \frac{x_{1}}{1-x_{4}}, \frac{x_{2}}{1-x_{4}}\right)
$$

is an alternative stereographic projection of $\mathbb{S}_{2}^{3}$ by the Lemma 4.1 and Definition 4.2.

Now, we give an example for spacelike AdS curve and its $W$-direction curve. Also, we show that its $W$-direction curve is a helix if the base curve is a helix. Besides, we draw pictures of these curves under the related stereographic projection by using Mathematica. 
Example 4.1. Let a spacelike AdS curve $\gamma$ be

$$
\gamma(s)=\left(\sqrt{3} \cosh (\sqrt{3} s), 3^{1 / 4} \cosh (2 s)+\sqrt{2+\sqrt{3}} \sinh (2 s), \sqrt{3} \sinh (\sqrt{3} s), \sqrt{2+\sqrt{3}} \cosh (2 s)+3^{1 / 4} \sinh (2 s)\right) .
$$

Then the geodesic curvatures of $\gamma$ are

$$
\kappa_{g}=\sqrt{2}, \tau_{g}=6 \sqrt{3},
$$

by straightforward calculations. Thus, $\gamma$ is a helix in $\mathbb{H}_{1}^{3}$ (see Figure 1.a). Also, $W$-direction curve of $\gamma$ is given by

$$
\widetilde{\gamma}(\widetilde{s}(s))=\left(\sqrt{2} \cosh (\sqrt{3} s), \sqrt{\frac{3}{2}}\left(3^{1 / 4} \cosh (2 s)+\sqrt{2+\sqrt{3}} \sinh (2 s)\right), \sqrt{2} \sinh (\sqrt{3} s), \sqrt{\frac{3}{2}}\left(\sqrt{2+\sqrt{3}} \cosh (2 s)+3^{1 / 4} \sinh (2 s)\right)\right)
$$

which is a timelike $\mathrm{dS}$ curve in $\mathbb{S}_{2}^{3}$ and its geodesic curvatures are

$$
\widetilde{\kappa}_{g}=\frac{\sqrt{23}}{6}, \widetilde{\tau}_{g}=-\frac{1}{23 \sqrt{3}},
$$

by straightforward calculations. Therefore, $\widetilde{\gamma}$ is also helix in $\mathbb{S}_{2}^{3}$ (see Figure 1.b).

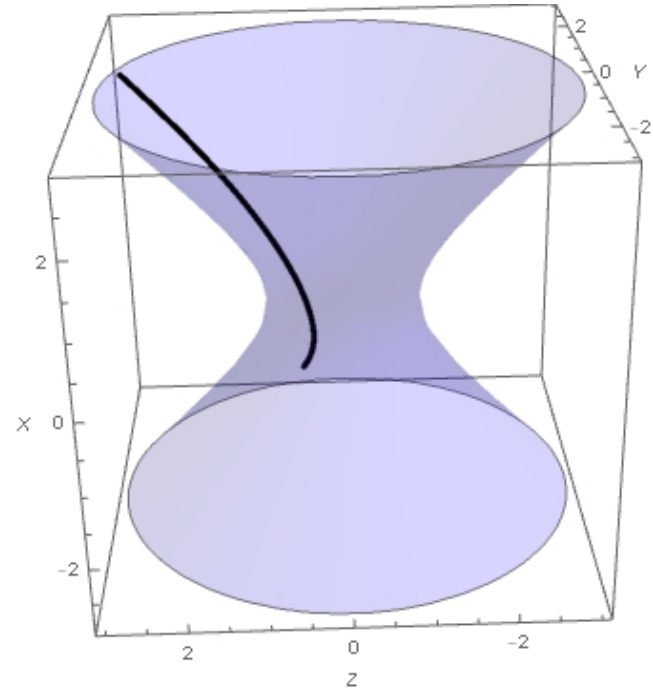

(a)

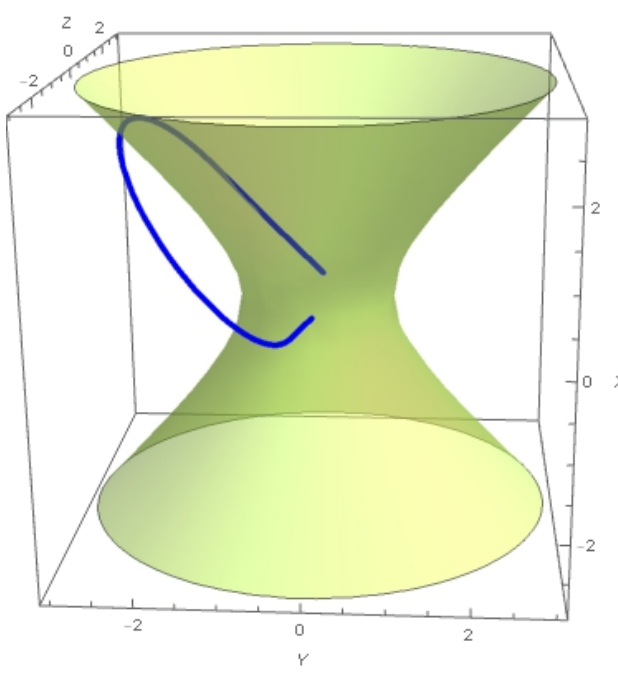

(b)

Figure 1. (a) The spacelike AdS curve $\gamma$ (b) The timelike $W$-direction dS curve of $\gamma$

\section{Conclusions}

In this paper, we define a special associated curve of non-degenerate base curve according to the Sabban apparatus in $\mathbb{H}_{1}^{3}$. Especially, we consider a $W$-direction curve whose $W=\frac{\boldsymbol{n}^{\prime}}{\left\|\boldsymbol{n}^{\prime}\right\|}$ of the base curve. Similarly, it can be considered the other special direction curves such as the vector field $W$ is either $\frac{\boldsymbol{t}^{\prime}}{\left\|\boldsymbol{t}^{\prime}\right\|}$ or $\frac{\boldsymbol{b}^{\prime}}{\left\|\boldsymbol{b}^{\prime}\right\|}$. As another point of view, $\frac{n^{\prime}}{\left\|n^{\prime}\right\|}$-direction curve corresponds to timelike(spacelike) principal normal image in $\mathbb{H}_{1}^{3}\left(\mathbb{S}_{2}^{3}\right)$ of the base curve. In this sense, $\frac{t^{\prime}}{\left\|t^{\prime}\right\|}$-direction curve and $\frac{b^{\prime}}{\left\|b^{\prime}\right\|}$-direction curve correspond to tangent image and binormal image in $\mathbb{M}^{3}\left(\widetilde{\delta}_{0}\right)$, respectively.

\section{Acknowledgements}

This work was supported by the Ahi Evran University Scientific Research Projects Coordination Unit. Project Number: FEF.A3.16.021 


\section{References}

[1] Barros, M., Ferrández, A., Lucas, P., Meroño, M. A., General helices in the three-dimensional Lorentzian space forms. Rocky Mountain J. Math. 31 (2001), no. 2, 373-388.

[2] Chen, L., Izumiya, S., Pei, D., Saji, K., Anti de Sitter horospherical flat timelike surfaces. Sci. China Math. 57 (2014), no. 9, 1841-1866.

[3] Choi, J. H., Kim, Y. H., Associated curves of a Frenet curve and their applications. Appl. Math. Comput. 218 (2012), no. 18, 9116-9124.

[4] Choi, J. H., Kim, Y. H., Ali, A. T., Some associated curves of Frenet non-lightlike curves in $\mathbb{E}_{1}^{3}$. J. Math. Anal. Appl. 394 (2012), no. 2, 712-723.

[5] Karlığa, B., On the generalized stereographic projection. Beiträge Algebra Geom. 37 (1996), no. 2, 329-336.

[6] Kızıltuğ, S., Önder, M., Associated curves of Frenet curves in three dimensional compact Lie group. Miskolc Math. Notes 16 (2015), no. 2, 953-964.

[7] Liu, H., Curves in Three Dimensional Riemannian Space Forms. Results in Mathematics 66 (2014), no. 3, 469-480.

[8] Macit, N., Düldül, M., Some new associated curves of a Frenet curve in $\mathbb{E}^{3}$ and $\mathbb{E}^{4}$. Turkish J. Math. 38 (2014), no. 6, 1023-1037.

[9] Mak, M., The Generalized Stereographic Projection and Inversion. Master's thesis, Gazi University Institute of Science and Technology, 2008.

[10] O'Neill, B., Semi-Riemannian Geometry with Applications to Relativity. Academic Press, New York, 1983.

\section{Affiliations}

MAHMUT MAK

AdDress: Ahi Evran University, Dept. of Mathematics, 40100, Kırşehir- Turkey.

E-MAIL:mmak@ahievran.edu.tr

HASAN ALTinbaş

AdDress: Ahi Evran University, Dept. of Mathematics, 40100, Kırşehir- Turkey.

E-MAIL: hasan.altinbas@ahievran.edu.tr 